\title{
Der Psychosoziale Krisendienst (PSKD) in Frankfurt am Main
}

ist ein Baustein des psychosozialen und sozialpsychiatrischen Hilfesystems der Mainmetropole. Er ist seit 1994 Teil des gemeindepsychiatrischen Hilfeangebotes in Frankfurt am Main. Der telefonische Dienst wurde für jene Menschen ins Leben gerufen, die sich in einer psychosozialen Krisensituation befinden und eine Anlaufstelle außerhalb der regulären Dienstzeiten der ambulanten Hilfeangebote suchen. Der Psychosoziale Krisendienst informiert, leistet kurzfristige Hilfen und vermittelt lösungsorientiert in das des psychiatrische Hilfesystems vor Ort.

Der Psychosozialer Krisendienst in Frankfurt am Main richtet sich speziell an den Personenkreis der psychisch kranken und seelisch behinderten Menschen, deren Partner und Angehörige sowie Personen aus dem sozialen Umfeld und diejenigen, die in die Situation involviert sind. Er soll Ratsuchenden helfen, bereits im Vorfeld psychosoziale Krisen zu bewältigen und der Eskalation von Problemsituationen vorzubeugen. Auf Wunsch wird die Anonymität des Hilfesuchenden gewahrt.

Den Psychosozialen Krisendienst erreichen oft Anrufe von psychisch kranken Menschen, die zwar gut in das ambulante Hilfesystem integriert sind, die aber außerhalb der Dienstzeiten, gerade an Wochenenden, in krisenhafte Situationen geraten, die aus eigener Kraft nicht mehr bewältigt werden können. In solchen Fällen leistet der Psychosoziale Krisendienstes Entlastungsgespräche, die lebenswichtig sein können. Ebenso erreichen den Krisendienst Anrufe von Menschen, die nach einem stationären Aufenthalt in einer Psychiatrie sich in ihrem häuslichen Umfeld (noch) nicht zu Recht finden und Krisen durchleben. Oft können solche problematischen Situationen durch ein stützendes und strukturierendes Gespräch aufgefangen werden. Notwendig ist hier, über das sozialpsychiatrische Hilfesystem zu informieren, zusammen mit der Empfehlung, am nächsten Tag die zuständige Psychosoziale Kontakt- und Beratungsstelle aufzusuchen. In den Anrufen wird oft die Verunsicherung, Angst, Hilflosigkeit, Wut oder Verzweiflung der Anrufenden spürbar. Durch Entlastungsgespräche und durch Angebote von weiterführenden Hilfen können Eskalationen der Problemlagen vorgebeugt und verhindert werden.

Die Finanzierung des Angebots erfolgt durch einen Zuschuss der Stadt Frankfurt am Main. Der Psychosoziale Krisendienst besteht aus den drei Organisationseinheiten Koordination und Organisation, Krisendienst im Einsatz und medizinischer Krisendienst:

- Die Koordination und Organisation des Krisendienstes wird durch die Bürgerhilfe Sozialpsychiatrie Frankfurt am Main e. V. sichergestellt. Sie erstellt quartalsweise die Dienstplanung und erledigt die daraus folgenden Aufgaben. Sie ist zudem im Einvernehmen mit dem Stadtgesundheitsamt für die konzeptionelle Weiterentwicklung des Psychosozialer Krisendienstes sowie für die Einhaltung der fachlichen Standards verantwortlich. Für die Koordination stellt die Bürgerhilfe 40 Prozent einer Vollzeitstelle sowie Ressourcen der Geschäftsstelle zur Verfügung. Sie ist weiterhin verantwortlich für die Finanzabwicklung, Dokumentation, Werbung neuer Mitarbeitenden, Organisation von Fortbildungen und Supervision, Planung der vierteljährlichen Teambesprechungen und die Auswertung von Statistiken. Zu den weiteren Aufgaben der Koordination gehört die Mitwirkung im gemeindepsychiatrischen Verbund in Frankfurt am Main. Daraus folgt die Vernetzungsarbeit mit allen Angeboten und Einrichtungen des ambulanten Versorgungssystems, niedergelassenen Fachärzten, städtischen Dienststellen, übergreifenden Hilfeangeboten, Kooperation mit den psychiatrischen Kliniken.
- Der Krisendienst im Einsatz wird von zirka 30 fachlich qualifizierten Mitarbeiterinnen und Mitarbeitern (Sozialarbeiter, Sozialpädagogen, Fachkrankenpfleger, teilweise mit therapeutischer Zusatzausbildung) sicher gestellt. Die Mitarbeitenden verfügen über Berufserfahrung in der Psychiatrie oder im Beratungsbereich. Die Telefongespräche werden mittels Rufumleitung in der häuslichen Umgebung der Krisendienstarbeiter durchgeführt. Der Psychosoziale Krisendienst in Frankfurt am Main ist durchgängig an 365 Tagen dienstbereit. Die Zeiten sind montags bis freitags von 17.00 bis $1.00 \mathrm{Uhr}$, am Wochenende und an Feiertagen von 9.00 bis 1.00 Uhr. Schwerpunktmäßig wird der Dienst an den Sonn- und Feiertagen genutzt. Die Krisenarbeiter werden mit einer Vielfalt von Anliegen und Problemen, Störungen und Lebenssituationen konfrontiert. Auch gänzlich unterschiedliche Arten von Hilfen sind gefordert: Das Spektrum reicht von lebenspraktischer Hilfe und Information über therapeutisch orientierte Hilfe bis hin zur Einleitung und Organisation von Notfallhilfen.

- Der medizinische Krisen- und Notfalldienst wird von den an der psychiatrischen Pflichtversorgung beteiligten Kliniken oder deren psychiatrischen Institutsambulanzen übernommen.

Einige Fallbeispiele können zeigen, mit welch unterschiedlichen Problemlagen der Psychosoziale Krisendienst konfrontiert wird:

- Anruferin mit Selbstverletzungsabsichten (»Schneidedruck «): Sie sei drei Monaten in stationärer Behandlung gewesen und wäre nicht arbeitsfähig. Sie habe Zukunftsängste, sei einsam. Sie habe finanzielle Probleme und verfüge kaum über soziale Kontakte. Die Anruferin wird durch den Mitarbeiter des Psychosozialen Krisendienst nach einem strukturierenden Entlastungsgespräch an die zuständige psychosoziale Beratungsstelle verwiesen. Die Vermittlung kam durch einen niedergelassenen Psychiater zustande.

- Die Mutter einer psychisch kranken Tochter fühlt sich mit der Problematik überfordert. Sie sei selbst gesundheitlich angeschlagen. Die Anruferin wird durch den Mitarbeiter des Psychosozialen Krisendienst nach einem Entlastungsgespräch an die zuständige psychosoziale Beratungsstelle vermittelt. Die Vermittlung kam durch die kirchliche Seelsorge zustande.

- Eine Frau, deren Ehemann sich suizidiert hat, macht sich große Vorwürfe. Sie fühle sich schuldig und verantwortlich. weil sie ihn nicht überwacht und den Suizid verhindert habe. Sie habe sich nun ganz aus sozialen Kontakten zurückgezogen. Da sie eine Beratung außerhalb des Psychosozialen Krisendienstes ablehnt, bleibt nur die Möglichkeit eines telefonischen Entlastungsgespräches.

- Anruferin mit Borderline-Persönlichkeitsstörung; wird betreut durch eine psychiatrische Institutsambulanz. Sie habe gerade die Trennung von ihrem Freund vollzogen. Fühle sich stark depressiv. Die Mitarbeiterin des Psychosozialen Krisendienst leistet Krisenintervention durch ein stützendes Gespräch.

- Anruferin fühle sich verfolgt und ausgenutzt. Habe keine Freunde. Eine psychosomatische Kur sei geplant. Sie sei alleine, gereizt und angespannt. Schimpft unaufhörlich. Im Laufe des Telefongesprächs wird die Anruferin ruhiger und entspannter. Es wird empfohlen, sich eine weitere Unterstützung in der psychosozialen Beratungsstelle zu holen.

Klaus Gerold

Klaus Gerold ist Sozialarbeiter bei der Bürgerhilfe

Sozialpsychiatrie Frankfurt am Main e. V.

Internet http://www.bsf-frankfurt.de 